\section{P138 DEVELOPMENT OF A TARGETED METABOLOMIC URINE- BASED PANEL FOR INFLAMMATORY BOWEL DISEASE}

1,2Kate Gallagher, ${ }^{1,2}$ Shiva T Radhakrishnan*, 'Jia V Li, 'Mark R Thurz, 1,3Elaine Holmes, ${ }^{1,2}$ Horace RT Williams. ${ }^{1}$ Imperial College London, London, UK; ${ }^{2}$ Imperial College Healthcare NHS Trust, London, UK; ${ }^{3}$ Australian National Phenome Centre, Perth, Australia

10.1136/gutjnl-2020-bsgcampus.213

Introduction Ulcerative Colitis (UC) and Crohn's Disease (CD), the Inflammatory Bowel diseases (IBD) are characterised by chronic relapsing remitting inflammation of the gastrointestinal tract. Over the past decade, metabolomic research has allowed for the characterisation of UC, CD, inactive and active disease; many biofluids have been analysed. However, findings have been inconsistent and few offer clinically useful quantitative data.

Methods A comprehensive literature review, and expansion of appropriate biochemical pathways, was carried out to identify metabolic targets for development into a fully quantifiable and targeted assay. A selection of these metabolites was validated for use in predicting IBD via a combination of univariate and multivariate techniques in proton nuclear magnetic resonance $\left({ }^{1} \mathrm{H}-\mathrm{NMR}\right)$ spectroscopy acquired urine data (IBD $=215$, controls $=101)$. A finalised panel was taken forward for an ultra-high-performance liquid chromatography-mass spectrometry (UHPLC-MS) urine-based assay development and consequent validation in a clinical cohort.

Results 42 metabolites were identified as discriminatory, for characterising IBD compared to controls: these have been taken forward for urinary assay development and subsequent validation. Prior analysis of urinary data from a heterogenous IBD cohort demonstrated that using a specified panel of metabolites, as opposed to all data acquired, considerably improved the predictive score (Q2), and significance of models generated (from $p=1$ to $p=0.003$ respectively). These metabolites were both of human and microbial origin, reiterating the importance of the gut microbiome in IBD. Following development, the panel will be validated on a de novo clinical cohort of IBD patients to quantify metabolic perturbations invoked by disease pathogenesis.

Conclusions This work highlights the efficacy of targeted metabolomic methods and the capabilities of these approaches to characterise IBD. The identification of refined metabolic changes with a pathway-driven approach will not only improve knowledge of both host and microbial metabolism in IBD but may also elucidate potential novel therapeutic targets and markers for therapeutic response.

\section{P139 MICROSCOPIC COLITIS: LOST OPPORTUNITY FOR DIAGNOSIS AND TREATMENT RESULTS IN HOSPITAL ADMISSIONS}

Suneil A Raju*, Thean S Chew, David S Sanders. Academic Unit of Gastroenterology, Sheffield, UK, Sheffield, UK

\subsection{6/gutjnl-2020-bsgcampus.214}

Background Microscopic colitis (MC) is a histological diagnosis. MC significantly affects patients' quality of life but there is limited data on the length of symptoms prior to diagnosis and the patient journey. We therefore evaluated the largest UK clinical data set on patients with MC.
Methods A UK study from 2 hospitals of retrospectively collected data from case notes between 2007 and 2017 of all patients diagnosed with histologically confirmed MC. Further data was collected on all colonoscopies complete for chronic diarrhoea/Irritable Bowel Type Syndrome (IBS) type symptoms in the same study period.

Results A total of 562 patients were diagnosed with MC of which $24.9 \%$ collagenous colitis $(72.1 \%$ female), $70.1 \%$ lymphocytic colitis $(63.2 \%$ female) and 5\% undifferentiated $\mathrm{MC}$ (75\% female). Collagenous colitis was diagnosed later than lymphocytic colitis (67 vs 63 years $(p=0.002)$

Patients reported symptoms prior to referral of 3 months (IQR 2 month-1 year). However, 15\% had symptoms 3-20 years. From primary care $56.0 \%$ were referred routinely and $44.0 \%$ via two week wait pathways $(2 \mathrm{ww})$. Patients under $2 \mathrm{ww}$ were seen quicker $(12$ days vs 24 days, $\mathrm{p}<0.0005)$ as were older patients $(\mathrm{p}<0.005)$.

Hospital admission was required in $6.4 \%$ of patients due to diarrhoea $(80.6 \%)$, acute kidney injury $(27.8 \%)$, abdominal pain $(16.7 \%)$ and collapse (5.6\%). 1.4\% of patients were admitted for other reasons and MC diagnosed incidentally. Older patients were more likely to be admitted (OR: 1.074, CI: 1.0 .4 - 1.1) and no patients were on budesonide prior to admission. The average length of stay was 12 days (IQR:8-21 days). Of patients admitted, $11.1 \%$ had had previous colonoscopies.

In the same study period 10,015 lower gastrointestinal endoscopies $(84.3 \%$ colonoscopies and $15.7 \%$ flexible sigmoidoscopies) were performed $(59.3 \%$ female, 57 years, IQR 4369 years). Colonoscopies were performed for investigation of chronic diarrhoea, IBS-diarrhoea (IBS-D), IBS-mixed (IBS-M), or suspected IBD $(22.4 \%, 59.0 \%, 14.6 \%$ and $3.9 \%)$. In total, $19.5 \%$ of colonoscopies conformed to biopsy guidelines. In the other cases biopsies were taken from incorrect sites: only left or right sides of the colon, the rectum and randomly (15.8\%, $10.7 \%, 24.2 \%$, and $58.7 \%$, respectively). In $8.6 \%$ of colonoscopies, no biopsies were taken. The highest adherence to guidelines $(48 \%)$ occurred in a subgroup of IBD where the indication was to rule out MC.

Conclusion This is the largest international study to report MC findings. Our data demonstrates lost opportunities for diagnosis and treatment of MC both in primary and secondary care which may ultimately result in hospital admission. This may suggest a limited understanding to $\mathrm{MC}$ from both primary and secondary care.

\section{P140 HETEROGENEITY IN OUTCOME ASSESSMENT FOR IBD IN ROUTINE PRACTICE: A MIXED-METHODS STUDY OF ENGLISH HOSPITALS}

Violeta Razanskaite*, Constantinos Kallis, Bridget Young, Paula Williamson, Keith Bodger. University of Liverpool, UK

\subsection{6/gutjnl-2020-bsgcampus.215}

Introduction Global initiatives have sought to standardise outcome assessment for inflammatory bowel disease (IBD) for clinical trials (Core Outcome Sets) and routine practice (ICHOM). Our aims were to investigate variability in the assessment and recording of outcomes elicited by clinicians from patients in hospital-based IBD outpatient services and to 
explore the contribution of patient, practitioner and hospital characteristics.

Methods We performed structured observations of consultations conducted with 102 patients by 24 clinicians at six hospitals across the North West region of England and analysis of electronic health records (EHR) generated for 909 previous consultations with the same patients. Audio-recordings of observed consultations and the EHR were analysed for a pre-specified checklist of 38 outcomes, including items required for clinical IBD activity indices and patient reported outcomes derived from Crohn's Disease Activity Index and Mayo score (PRO2/PRO3). We performed 24 semi-structured interviews with the clinicians to explore views on structured, standardised outcome assessment in routine practice.

Results Of the 38 pre-specified clinical outcomes, a median of 11.5 (IQR: 8-13.25) were elicited in observed consultations (Ob), but only 6 (3-9) recorded in EHR. There was substantial inter- and intra-individual variation in the number of outcomes captured per consultation by clinicians (mean range per practitioner: 6.6 in $\mathrm{Ob}$; 9.2 in EHR) and for patients (mean range per patient: 9.8 in EHR). In multivariate analysis, a diagnosis of Crohn's disease $(\beta$ coef $0.89, \mathrm{p}=0.003)$, management changes $(\beta$ coef $0.67, \mathrm{p}=0.001)$ and nurse-led consultations ( $\beta$ coef $3.24, p<0.001$ ) were significantly associated with a higher number of outcomes recorded in EHR. Disease activity indices were not collected routinely (only 15\% Ob; 9\% EHR), but coverage of $\mathrm{PRO} 2$ and $\mathrm{PRO} 3$ items was more common (Ob v R: PRO2: 80\% v 60\% for UC; 76\% v 44\% for CD; PRO3: $76 \%$ v 27\% for CD). Standardised quantification of symptoms as defined by activity indices was rare. Clinicians reported a range of logistical and IT barriers to standardisation.

Conclusions Substantial variation was observed in the elicitation and recording of IBD outcomes, with information loss between consultation and capture in EHR. Variation was not readily explained by patient, practitioner or hospital characteristics although the number of outcomes recorded were significantly higher for CD, for consultations with nurses and for consultations involving management changes. Clinicians express varied viewpoints on the merits of standardisation versus individualised clinical narratives. These findings suggest clinical trials do not reflect the process of day-to-day decision-making. Direct capture of patient reported outcomes may provide a more feasible approach to standardisation of outcomes assessment.

\section{P141 DIFFERENT FAILURE RATES AFTER NON-MEDICAL SWITCHING 744 PATIENTS FROM ADALIMUMAB ORIGINATOR TO 2 ADALIMUMAB BIOSIMILARS}

\begin{abstract}
${ }^{1}$ Denise Rosembert ${ }^{*},{ }^{2}$ Anshuman Malaviya, ${ }^{2}$ Jane How, ${ }^{3}$ Fiona Toh, ${ }^{3}$ Jacqueline Tomlinson, ${ }^{4}$ Allison Nightingale, ${ }^{4}$ Cristina Garcia-Sueiro, ${ }^{4}$ Pippa Herbert, ${ }^{4}$ Julien Magsino, ${ }^{1}$ Maria Roe, ${ }^{2}$ Deepak Jadon, ${ }^{3}$ Paul Norris, ${ }^{4}$ Tim Raine. ${ }^{1}$ Pharmacy Department, Cambridge University Hospitals, Cambridge, UK; ${ }^{2}$ Rheumatology Department, Cambridge University Hospitals, Cambridge, UK; ${ }^{3}$ Dermatology Department, Cambridge University Hospitals, Cambridge, UK; ${ }^{4}$ Gastroenterology Department, Cambridge University Hospitals, Cambridge, UK
\end{abstract}

\subsection{6/gutjnl-2020-bsgcampus.216}

Introduction Biosimilar adalimumab became available in the UK in October, 2018, with payers introducing a series of measures to drive the adoption of the 'best value' adalimumab $^{1}$. Consideration of value should include risk of loss of efficacy and adverse reactions, which might drive further biologic switches or 'reverse switches' to originator. Managing potential 'nocebo effect' requires high levels of patient engagement, whilst the presence or absence of citrate buffer may affect patient reported discomfort, hence patient treatment perceptions.

Method Patients were switched to 2 different biosimilar adalimumabs, citrate containing (ADA1) and citrate-free (ADA2). The choice of biosimilar was made for clinical and economic reasons. Patients were informed of the switch to either ADA1 or ADA2 in advance via letter and at clinic visits. Patients who reported problems after switching were reviewed.

Results 744 patients were switched from originator adalimumab (ADA0) to ADA1 or ADA2. Rheumatology and dermatology patients were switched to ADA1, whilst gastroenterology patients were switched to ADA2, except for those with a latex allergy, where ADA1 was used (or ADA2 in a latex-free syringe). A total of $49(6.6 \%)$ patients reported problems and a further switch of drug was judged necessary in 48 (table 1). Switch failure (SF) was significantly more likely with ADA1 than ADA2 $(7.7 \%$ vs $1.8 \% ; p=0.006$ ). Median time to $S F$ was 97 days (range 5-196 days). The commonest reason for SF was disease flare (23 patients, 48\%), followed by injection site pain or reaction (21 patients, 44\%). Disease flare and injection site problems were more likely to be reported with ADA1 than ADA2 (flare: $23 / 583$ vs $0 / 161, p=0.008$; injection site pain $20 / 583$ vs $1 / 161, p=0.06)$. For patients reporting disease flare, clinicians tended to select a reverse switch to ADA0, whilst for those with injection site problems, a further switch to a different biosimilar was the preferred approach; both strategies were effective at recapture.

Abstract P141 Table 1 Biosimilar further switches and reverse switches by 1 st biosimilar and by therapeutic area

\begin{tabular}{lllll}
\hline & $\begin{array}{l}\text { ADA1 to } \\
\text { ADA0 (reverse } \\
\text { switch) }\end{array}$ & $\begin{array}{l}\text { ADA1 to } \\
\text { ADA2 (further } \\
\text { switch) }\end{array}$ & $\begin{array}{l}\text { ADA2 to } \\
\text { ADA0 (reverse } \\
\text { switch) }\end{array}$ & $\begin{array}{l}\text { ADA2 to } \\
\text { ADA1 (further } \\
\text { switch) }\end{array}$ \\
\hline Rheumatology & $28 / 512(5.5 \%)$ & $14 / 512(2.7 \%)$ & $0 / 0$ & $0 / 0$ \\
Dermatology & $1 / 64(1.6 \%)$ & $2 / 64(3.1 \%)$ & $0 / 0$ & $0 / 0$ \\
Gastroenterology & $1 / 7(14 \%)$ & $0 / 0$ & $2 / 161(1.2 \%)$ & $1 / 161(0.6 \%)$ \\
TOTAL & $30 / 583(5.1 \%)$ & $15 / 583(2.6 \%)$ & $2 / 161(1.2 \%)$ & $1 / 161(0.6 \%)$ \\
Total switch & ADA1 switch failures: $45 / 583$ & ADA2 switch failures: $3 / 161$ \\
failures & $(7.7 \%)$ & & $(1.8 \%)$ & \\
\hline
\end{tabular}

Conclusion In the context of an effective patient communication strategy, adalimumab biosimilar switching was associated with overall low rates of patient-reported problems in a large cohort. Despite an identical communication strategy, significantly higher rates of injection site problems and disease flares were seen between 2 different biosimilars, reflected in a higher need for further switches. These differences may impact cost effectiveness of any chosen strategy.

\section{REFERENCE}

1. NHSE Biosimilar medicines https://www.england.nhs.uk/medicines/biosimilar-medicines/cited 14.11.2019 